\title{
Optimización de la resistencia a compresión de lechadas de cemento reforzadas con aditivos
}

\section{Optimization of compressive strength in admixture-reinforced cement-based grouts}

\author{
ö. $\operatorname{Tan}^{(*)}$ y A. Sahin Zaimoglu(**)
}

Recepción/Received: 1-IV-05

Aceptación/Accepted: 26-XII-07

RESUMEN

En el presente trabajo se ha intentado optimizar, mediante el método de Taguchi, las resistencias a compresión (a las edades de 7, 14 y 28 días) de lechadas de cemento reforzadas con bentonita, cenizas volantes y humo de sílice. Se diseñaron los experimentos de acuerdo con un arreglo ortogonal tipo $L_{16}$ en el que se contemplaban tres factores: la bentonita $(0,0,5,1$ y $3 \%)$, las cenizas volantes $(10,20,30$ y $40 \%)$ y el humo de sílice $(0,5,10$ y $20 \%)$ (porcentajes en peso del sólido). Los datos obtenidos se analizaron con mediante ANOVA y el método de Taguchi. De acuerdo con los resultados experimentales, el contenido tanto de cenizas volantes como de humo de sílice desempeña un papel significativo en la resistencia a compresión. Por otra parte, las condiciones óptimas que se han identificado son: $0 \%$ bentonita, $10 \%$ cenizas volantes, $20 \%$ humo de sílice y 28 días de tiempo de curado. La resistencia a compresión máxima conseguida en las anteriores condiciones era de 17,1 $\mathrm{MPa}$.

Palabras clave: lechada de cemento, bentonita, cenizas volantes, humo de sílice, método de Taguchi.
SUMMARY

The Taguchi method was used in this study to optimize the unconfined (7-, 14- and 28-day) compressive strength of cement-based grouts with bentonite, fly ash and silica fume admixtures. The experiments were designed using an $L_{16}$ orthogonal array in which the three factors considered were bentonite $(0 \%, 0.5 \%, 1.0 \%$ and $3 \%)$, fly ash (10\%, 20\%, 30\% and 40\%) and silica fume (0\%, 5\%, $10 \%$ and $20 \%$ ) content. The experimental results, which were analyzed by ANOVA and the Taguchi method, showed that fly ash and silica fume content play a significant role in unconfined compressive strength. The optimum conditions were found to be: $0 \%$ bentonite, 10\% fly ash, $20 \%$ silica fume and 28 days of curing time. The maximum unconfined compressive strength reached under the above optimum conditions was 17.1 MPa.

Keywords: cement based grouts, bentonite, fly ash, silica fume, taguchi method.

\footnotetext{
(*) Universidad de Selcuk, Konya (Konya, Turquía).

(**) Universidad de Ataturk, Erzurum (Erzurum, Turquía).
} 


\section{INTRODUCCIÓN}

Desde hace mucho tiempo se han utilizado las lechadas de cemento en aplicaciones geotécnicas ante problemas relacionados con la cimentación, para mejorar la capacidad resistente del suelo, reducir su permeabilidad y deformación, rellenar los huecos y construir muros pantalla. Además, en los últimos años el empleo de las lechadas de cemento se ha extendido al campo medioambiental, donde se utilizan para el sellado de residuos tóxicos o radioactivos de baja actividad (1). A fin de reducir costes o mejorar algunas de las propiedades de las mezclas utilizadas en el cemento inyectado, se incorporan al cemento materiales como las cenizas volantes (CV), el humo de sílice (HS), la bentonita (B) o el látex. Con la incorporación del látex, la lechada presenta mejoras de adherencia, impermeabilidad y resistencia a la flexotracción (2). Las CV son un subproducto industrial que resulta de la combustión del carbón pulverizado en las centrales térmicas. Su inclusión reduce los costes, disminuye la exudación y aumenta la densidad, mejorando tanto la impermeabilidad como la durabilidad de las lechadas. La B es una arcilla típica que se genera por la alteración de la ceniza volcánica. Reduce la exudación y mejora tanto las propiedades reológicas como la estabilidad de las lechadas. El HS es un polvo muy fino que se obtiene como subproducto de la fabricación de metales o aleaciones silíceos. Su extrema finura y alto contenido de silicio hacen que el HS sea una excelente puzolana. Disminuye la porosidad y la permeabilidad y aumenta la resistencia mecánica de las lechadas. Tan y Zaimoglu (3) han investigado la influencia en los tiempos de fraguado de las lechadas de cemento de la incorporación de las CV, el HS y la B. En ese trabajo, observaron que los tiempos de fraguado inicial y final disminuyeron al aumentar el HS y la B. Al investigar los efectos de los aditivos en la exudación de las lechadas de cemento, Tan et al. (4) constataron que mientras que ésta disminuye al aumentar el HS o la B, no se aprecian diferencias significativas al añadir cantidades crecientes de las CV.

Con el presente trabajo se pretende investigar la influencia de los tres aditivos (CV, HS y B) en la resistencia a compresión de lechadas a las edades de 7, 14 y 28 días. Se planteó el trabajo experimental de manera que pudieran identificarse, mediante el método de Taguchi, las condiciones óptimas de los factores que influyen en la resistencia a compresión. En la ref. (5) se recoge una descripción más detallada de dicho estudio.

\section{PROGRAMA EXPERIMENTAL}

\subsection{Materiales}

En la presente investigación se ha empleado cemento Portland (CEM I) fabricado por la empresa Set de Ankara (Turquía). El HS, las CV y la B procedían de Antalya Electro Metallurgy Enterprise, la central térmica de Cayırhan y la mina de Karakayalar, respectivamente. La composición química de los materiales se muestra en la Tabla 1. Todos los experimentos se realizaron de acuerdo con la norma ASTM C109 (6). La velocidad y tiempo de amasado eran de 1.000 rpm y cinco minutos, respectivamente. La relación agua/cemento era de 0,75 . Al agua de amasado se añadieron el cemento seco, previamente mezclado, las CV y el HS. Finalmente, se agregó una lechada de B $(10 \%$ B + $90 \%$ agua) a la hormigonera, estando ésta en funcionamiento durante todo el proceso de incorporación de los componentes. Los componentes se amasaron durante cinco minutos. Con la mezcla resultante se prepararon probetas cilíndricas de $38 \mathrm{~mm}$ x $76 \mathrm{~mm}$, que fueron desmoldadas a las 24 horas. Después del curado en condiciones normalizadas durante 7,14 y 28 días, las probetas se sometieron a la prueba de resistencia a compresión. Como referencia se utilizaron probetas de cemento sin aditivos, preparadas y probadas por el mismo procedimiento.

Tabla 1

Composición química de los materiales utilizados

\begin{tabular}{|c|c|c|c|c|}
\hline & Cemento (\%) & Bentonita (\%) & Cenizas volantes (\%) & Humo de sílice (\%) \\
\hline $\mathrm{SiO}_{2}$ & 19,80 & 60,75 & 47,5 & 90 \\
\hline $\mathrm{AI}_{2} \mathrm{O}_{3}$ & 5,61 & 18,9 & 15,95 & 2 \\
\hline $\mathrm{Fe}_{2} \mathrm{O}_{3}$ & 3,42 & 3,05 & 16,3 & 0,8 \\
\hline $\mathrm{CaO}$ & 62,97 & 2,75 & 6,6 & 1,0 \\
\hline $\mathrm{MgO}$ & 1,76 & 2,1 & 4,65 & 1,5 \\
\hline $\mathrm{SO}_{3}$ & 2,95 & - & 15,95 & - \\
\hline $\mathrm{Na}_{2} \mathrm{O}$ & 0,47 & 2,7 & - & 0,7 \\
\hline $\mathrm{K}_{2} \mathrm{O}$ & 0,87 & 0,95 & 0,86 & 0,8 \\
\hline Pérdida al fuego & 2,17 & - & 2,36 & 0,7 \\
\hline Densidad $\left(\mathrm{g} / \mathrm{cm}^{3}\right)$ & 3,08 & 1,04 & & 2,25 \\
\hline
\end{tabular}




\subsection{Metodología analítica de Taguchi}

En un diseño factorial completo el número de experimentos requerido puede ser muy alto, siendo prácticamente imposible efectuarlos todos. El sistema lineal desarrollado por Taguchi (7) ha resultado muy útil para los análisis científicos y técnicos. Este método supone una herramienta poderosa, sobre todo a la hora de diseñar un sistema robusto. Además, constituye un enfoque sistemático al problema de optimizar el diseño en experimentos dirigidos a determinar el rendimiento y la calidad. Para ello emplea objetivos de la respuesta o criterios basados en el cociente señal/ruido (S/R), cuya unidad es el $\mathrm{dB}(8)$. El método contempla tres criterios u objetivos: cuanto más grande mejor, cuanto menor mejor, y nominal es lo mejor. Dichos criterios se definen mediante las Ecuaciones [1]-[3]:

Cuanto más grande mejor:

$$
S />R=-10 \log _{10}\left(\frac{1}{n} \sum_{i=1}^{n} \frac{1}{Y_{i}^{2}}\right)
$$

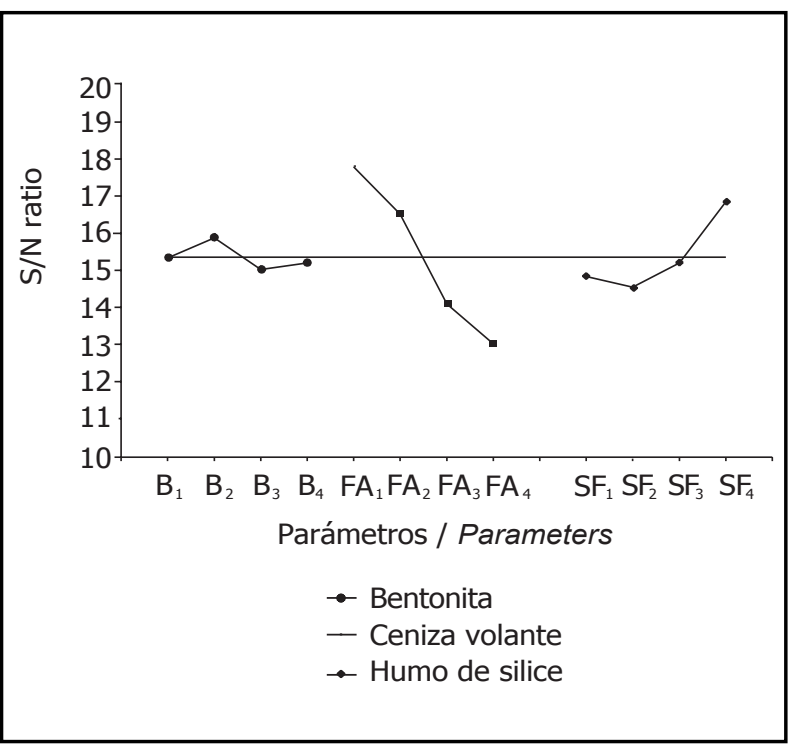

Figura 1. Cociente $S / R$ en función del nivel de los factores a los 7 días.
Cuanto menor mejor:

$$
S />R=-10 \log _{10}\left(\frac{1}{n} \sum_{i=1}^{n} \mathrm{Y}_{i}^{2}\right)
$$

Nominal es lo mejor:

$$
\left.S />R=-10 \log _{10}\left(\frac{1}{n} \sum_{i=1}^{n} \mathrm{Y}_{i}-\mathrm{Y}_{0}\right)^{2}\right)
$$

Siendo $\mathrm{n}$ el número de repeticiones de una combinación experimental, $Y_{i}$ el valor experimental e $Y_{0}$ el valor nominal deseado. Para el diseño de los experimentos se utilizan tablas normalizadas que se denominan arreglos ortogonales (AO). En el presente trabajo se han definido tres factores de cuatro niveles cada uno (Tabla 2). La Tabla 5 muestra el arreglo ortogonal empleado en los experimentos $\left(L_{16}\right)$. En un arreglo interno de tipo $L_{16}$, es preciso realizar un mínimo de 16 experimentos. El orden de éstos se ha obtenido mediante la incorporación, en las columnas del arreglo ortogonal, de los factores extraídos del diseño

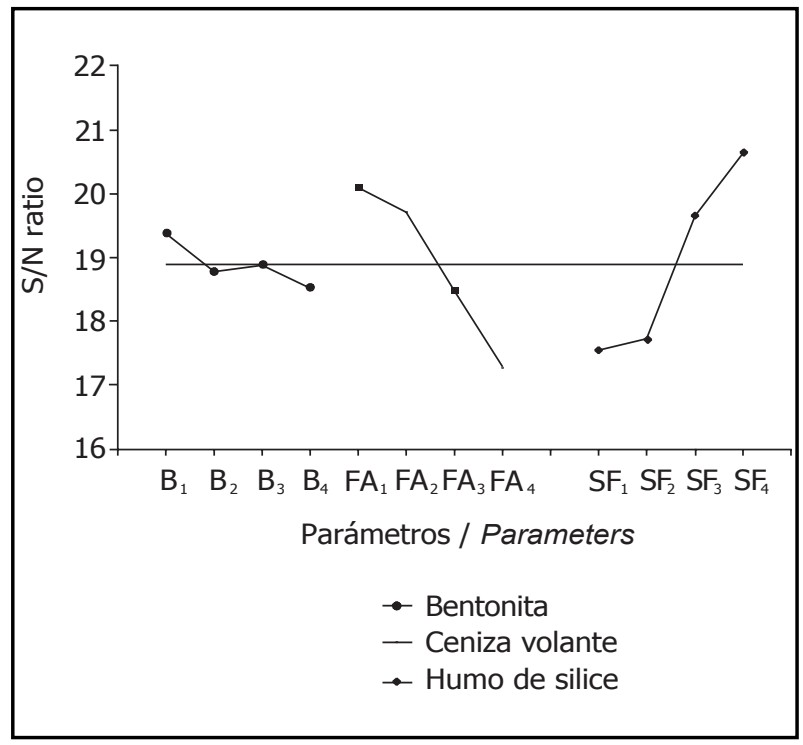

Figura 2. Cociente $S / R$ en función del nivel de los factores a los 14 días.

Tabla 2

Los tres factores con sus cuatros niveles estudiados en los experimentos

\begin{tabular}{|c|c|c|c|}
\hline \multirow{2}{*}{ Niveles } & \multicolumn{2}{|c|}{ Factores } \\
\cline { 2 - 4 } & $\begin{array}{c}\text { F1 } \\
\text { Bentonita (\%) }\end{array}$ & $\begin{array}{c}\text { F2 } \\
\text { Cenizas volantes }\end{array}$ & $\begin{array}{c}\text { F3 } \\
\text { Humo de sílice (\%) }\end{array}$ \\
\hline 1 & 0 & 10 & 0 \\
\hline 2 & 0,5 & 20 & 5 \\
\hline 3 & 1 & 30 & 10 \\
\hline 4 & 3 & 40 & 20 \\
\hline
\end{tabular}


experimental recogido en la Tabla 5. Para la optimización, se prepararon tres muestras por prueba. Los resultados se exponen en la Tabla 3. Los resultados de las pruebas de las muestras de sólo cemento se recogen en la Tabla 4. Los valores de S/R se calcularon para las probetas de 7, 14 y 28 días de acuerdo con el criterio "cuanto más grande mejor", a partir de la Ecuación [1]. Los valores se recogen en la Tabla 5. Los factores que maximizan la relación S/R estaban en su nivel óptimo. Según el método de Taguchi, es posible que el experimento que corresponde a las condiciones óptimas no se realice durante las pruebas. En estos casos, puede calcularse el intervalo de confianza (IC) para el nivel de error elegido a partir de la Ecuación [4].

$$
C I=\mu+\sqrt{\frac{F\left(1, n_{2}\right) V_{e}}{N_{e}}}
$$

donde $\mathrm{F}\left(1, \mathrm{n}_{2}\right)$ es el valor de $\mathrm{F}$ en el nivel correspondiente, que puede encontrarse en la tabla de la razón de varianzas o $\mathrm{F}$ de cualquier libro de estadística; $\mathrm{V}_{\mathrm{e}}$ es la varianza de error (según ANOVA) y $\mathrm{N}_{\mathrm{e}}$ es el número efectivo de réplicas. Las condiciones óptimas pueden predecirse mediante la determinación del punto de equilibrio del arreglo ortogonal, según la ecuación [5] (9).

$$
Y_{i}=\mu+X_{i}+e_{i}
$$

siendo $\mathrm{m}$ el promedio global del valor del rendimiento en las condiciones óptimas, $\mathrm{C}_{i}$ el efecto fijo de la combinación factor-nivel utilizado en la prueba y $\mathrm{e}_{i}$ el error aleatorio de cada prueba.

\section{RESULTADOS Y DISCUSIÓN}

En la Tabla 5 se exponen los cocientes S/R calculados al sustituir los resultados de las pruebas (Tabla 3 ) en la Ecuación [1]. El procedimiento para calcular el cociente $\mathrm{S} / \mathrm{R}$ puede explicarse mediante un ejemplo. El primer valor de $\mathrm{S} / \mathrm{R}$ a la edad de 7 días puede calcularse de la siguiente manera [6]:

$$
S / R=-10 \log _{10} \frac{1}{3}\left(\frac{1}{(8.27)^{2}}+\frac{1}{(7.50)^{2}}+\frac{1}{(8.38)^{2}}\right)=18.08
$$

Tabla 3

Resultados de las pruebas de resistencia a compresión

\begin{tabular}{|c|c|c|c|c|c|c|c|c|c|}
\hline \multirow{2}{*}{ Prueba } & \multicolumn{9}{|c|}{ Resistencia a compresión (MPa) } \\
\cline { 2 - 10 } & \multicolumn{3}{|c|}{$\mathbf{7}$ días } & \multicolumn{9}{c|}{$\mathbf{1 4}$ días } & \multicolumn{4}{c|}{ 28 días } \\
\hline & $\mathbf{1}$ & $\mathbf{2}$ & $\mathbf{3}$ & $\mathbf{1}$ & $\mathbf{2}$ & $\mathbf{3}$ & $\mathbf{1}$ & $\mathbf{2}$ & $\mathbf{3}$ \\
\hline 1 & 8,27 & 7,50 & 8,38 & 10,58 & 10,32 & 10,32 & 13,23 & 14,11 & 13,66 \\
\hline 2 & 5,73 & 6,17 & 6,17 & 7,50 & 7,15 & 7,50 & 10,14 & 10,58 & 10,73 \\
\hline 3 & 4,41 & 4,30 & 4,41 & 8,82 & 9,00 & 9,00 & 8,82 & 9,52 & 9,20 \\
\hline 4 & 5,73 & 5,29 & 5,56 & 10,2 & 11,30 & 11,45 & 12,17 & 12,17 & 13,23 \\
\hline 5 & 7,06 & 7,50 & 7,06 & 9,26 & 8,55 & 8,80 & 13,23 & 12,70 & 12,08 \\
\hline 6 & 6,17 & 6,40 & 6,61 & 8,20 & 8,60 & 9,08 & 9,70 & 9,70 & 9,70 \\
\hline 7 & 7,06 & 6,61 & 7,06 & 9,70 & 10,58 & 10,14 & 15,43 & 15,7 & 14,29 \\
\hline 8 & 5,18 & 4,63 & 4,41 & 7,76 & 7,23 & 7,23 & 8,82 & 8,38 & 8,82 \\
\hline 9 & 7,94 & 7,06 & 7,50 & 11,91 & 11,02 & 11,40 & 12,35 & 12,35 & 13,23 \\
\hline 10 & 7,50 & 7,06 & 7,50 & 12,21 & 12,17 & 12,17 & 12,78 & 13,23 & 14,29 \\
\hline 11 & 4,76 & 4,41 & 4,76 & 6,53 & 6,61 & 6,70 & 8,82 & 9,08 & 9,26 \\
\hline 12 & 3,97 & 3,97 & 3,97 & 6,35 & 6,35 & 6,79 & 8,38 & 7,94 & 8,11 \\
\hline 13 & 8,35 & 8,38 & 8,47 & 9,88 & 9,70 & 10,30 & 14,11 & 15,43 & 15,87 \\
\hline 14 & 7,06 & 7,28 & 7,06 & 11,29 & 11,29 & 11,29 & 14,20 & 13,05 & 15,26 \\
\hline 15 & 4,92 & 4,41 & 4,85 & 8,20 & 8,38 & 8,20 & 11,91 & 11,75 & 11,64 \\
\hline 16 & 3,79 & 3,92 & 3,97 & 5,56 & 5,47 & 5,29 & 6,00 & 6,00 & 6,00 \\
\hline
\end{tabular}

Tabla 4

Resistencia a compresión de las probetas de referencia

\begin{tabular}{|c|c|c|c|c|c|}
\hline \multirow{2}{*}{ Edad } & \multicolumn{5}{|c|}{ Resistencia a compresión (MPa) } \\
\cline { 2 - 6 } & $\mathbf{1}$ & $\mathbf{2}$ & $\mathbf{3}$ & $\mathbf{4}$ & Promedio \\
\hline 7 días & 9,08 & 8,82 & 7,23 & 8,82 & 8,5 \\
\hline 14 días & 14,73 & 15,17 & 16,76 & 15,34 & 15,5 \\
\hline 28 días & 18,96 & 19,40 & 17,64 & 17,64 & 17,41 \\
\hline
\end{tabular}


Tabla 5

Arreglo otorgal $\mathrm{L}_{16}$

\begin{tabular}{|c|c|c|c|c|c|c|}
\hline \multirow{2}{*}{ Prueba } & \multicolumn{3}{|c|}{ Factores y niveles } & \multicolumn{3}{c|}{ Cociente S/R } \\
\cline { 2 - 7 } & $\mathbf{B}$ & $\mathbf{F A}$ & $\mathbf{S F}$ & $\mathbf{7}$ días & $\mathbf{1 4}$ días & $\mathbf{2 8}$ días \\
\hline 1 & 1 & 1 & 1 & 18,08 & 20,34 & 22,70 \\
\hline 2 & 1 & 2 & 2 & 15,58 & 17,36 & 20,40 \\
\hline 3 & 1 & 3 & 3 & 12,81 & 19,03 & 19,24 \\
\hline 4 & 1 & 4 & 4 & 14,83 & 20,78 & 21,93 \\
\hline 5 & 2 & 1 & 2 & 17,14 & 18,94 & 22,04 \\
\hline 6 & 2 & 2 & 1 & 16,11 & 18,69 & 19,74 \\
\hline 7 & 2 & 3 & 4 & 16,77 & 20,10 & 23,58 \\
\hline 8 & 2 & 4 & 3 & 13,46 & 17,38 & 18,76 \\
\hline 9 & 3 & 1 & 3 & 17,47 & 21,16 & 22,02 \\
\hline 10 & 3 & 2 & 4 & 17,31 & 21,72 & 22,54 \\
\hline 11 & 3 & 3 & 1 & 13,32 & 16,41 & 19,13 \\
\hline 12 & 3 & 4 & 2 & 11,97 & 16,24 & 18,21 \\
\hline 13 & 4 & 1 & 4 & 18,48 & 19,96 & 23,57 \\
\hline 14 & 4 & 2 & 3 & 17,06 & 21,05 & 22,97 \\
\hline 15 & 4 & 3 & 2 & 13,46 & 18,34 & 21,41 \\
\hline 16 & 4 & 4 & 1 & 11,80 & 14,71 & 15,56 \\
\hline S/R promedio & & & & 15,35 & 18,89 & 20,86 \\
\hline
\end{tabular}

Los demás valores de $\mathrm{S} / \mathrm{R}$ pueden hallarse del mismo modo. En la Tabla 6 se muestra la influencia que ejercen sobre la resistencia a compresión los distintos niveles de cada uno de los factores a los 7, 14 y 28 días. Para el primer nivel del factor $B$, el valor promedio de $S / R$ puede calcularse a partir de la siguiente expresión [7]:

$$
S / R_{\text {average }}=\frac{1}{4}(18.08+15.58+12.81+14.83)=15.33
$$

Como se ve en la tabla, para las probetas de 7 días de edad, el valor promedio de S/R se maximiza en el nivel 1 del factor CV. En las muestras de 14 y 28 días, en cambio, el valor promedio mayor de S/R se consigue con el nivel 4 del factor HS. Los gráficos en que se representa la respuesta, es decir, que indican cómo los distintos factores influyen en la resistencia a compresión, se reproducen en las Figuras 1-3. Según se aprecia en la Figura 1 , a los 7 días el factor que influye de manera más notable en la resistencia a compresión es el aditivo CV. Al aumentar el porcentaje de dicho factor, disminuye la resistencia a compresión. El factor $\mathrm{B}$ apenas modifica la resistencia a compresión de las probetas de 7 días de edad. En cuanto al factor HS, cuando el porcentaje agregado es del $5 \%$, la resistencia a compresión de las muestras de 7 días disminuye, incrementándose cuando el porcentaje añadido es del 10 y del $20 \%$. En la Figura 2, que muestra el comportamiento de la resistencia a compresión a los 14 días, se observa una disminución de su valor al aumentar el porcentaje de los factores B y CV; ante mayores porcentajes del factor HS, en cambio, se produce una mejora de la resistencia a compresión importante. En la Figura 3, que representa los resultados a los 28 días, de nuevo se aprecia una disminución de la resistencia a compresión al aumentar el porcentaje del factor CV. De modo similar, la resistencia a compresión también crece con el porcentaje del factor HS añadido a la mezcla.

Se realizó un análisis de la varianza (ANOVA) de los datos relativos a la influencia ejercida por los factores $B$, CV y HS sobre la resistencia a compresión de las probetas de 7, 14 y 28 días de edad. Los resultados de dicho análisis se resumen en la Tabla 7 , que muestra que el factor que produce el mayor aumento de la resistencia a compresión a los siete días es el aditivo CV. La aportación del factor CV $(75,2 \%)$ es mayor, con diferencia, que la del factor HS (16,8\%). Por el contrario, a los 14 días la contribución de HS (44,3\%) es mayor que la de CV (31,8\%). Pero de nuevo a los 28 días la contribución de CV $(43,6 \%)$ es mayor que la de HS $(35,7 \%)$. En todos los casos, la aportación de $\mathrm{B}$ es despreciable.

Las condiciones óptimas determinadas para maximizar la resistencia a compresión ("cuanto más grande mejor") quedan recogidas en la Tabla 8 . Se determinaron igualmente los valores previstos y los intervalos de confianza al $90 \%$. Se realizaron pruebas de corroboración en las condiciones determinadas como óptimas, cuyos resultados se exponen en la Tabla 8. De acuerdo con ella, el resultado experimental para las probetas de 7 días se aproximaba a 9,25 MPa cuando se utilizaba 0,5\% B, 
$10 \%$ CV y $20 \%$ HS. Sin embargo, a los 14 y 28 días, la combinación con la que se obtuvieron resultados próximos a 12,2 y 17,1 , respectivamente, consistía en $0 \% B$, $10 \%$ CV y $20 \%$ HS. En la Figura 4 se muestran los valores de la resistencia a compresión de las probetas de referencia (de sólo cemento $(\mathrm{C})$ ), preparadas con los porcentajes óptimos de los componentes (O) y realizando 16 pruebas) a los 7, 14 y 28 días. Según esta figura, la utilización de materiales adicionales en las lechadas alcanza los mismos valores de resistencia a compresión, siendo estas mezclas muy económicas.

Según se desprende de la evaluación, la influencia del factor B no es relevante para las mezclas utilizadas en el cemento inyectado, en las que la relación líquido/sólido $=0,75$. Por otra parte, la incorporación de las CV disminuye la resistencia a compresión, que aumenta al añadir el HS. Estos resultados concuerdan con los de investigaciones anteriores $(1,10,11)$.
La influencia de los aditivos en la resistencia a compresión de lechadas de cemento puede explicarse por el comportamiento de dichos materiales, en el que pueden distinguirse dos mecanismos: por una parte, la reacción puzolánica; y por otra, el efecto "filler". En la reacción puzolánica, estos aditivos reaccionan con el hidróxido de calcio que se produce al hidratarse el cemento. En el efecto "filler", las partículas del aditivo rellenan los huecos microscópicos entre los granos de cemento.

Las $\mathrm{CV}$ de la clase $\mathrm{F}$ (contenido de $\mathrm{SiO}_{2}+\mathrm{Al}_{2} \mathrm{O}_{3}+\mathrm{Fe}_{2} \mathrm{O}_{3} \geq 70 \%$ y contenido de $\mathrm{CaO}<10 \%)$ son sólo puzolánicas, careciendo prácticamente de valor cementante. Sin embargo, son capaces de reaccionar químicamente con el hidróxido de calcio a temperatura ambiente para formar compuestos con propiedades cementantes. Según los resultados de trabajos anteriores, las CV reducen la resistencia a compresión del hormigón en las edades iniciales. No obstante, a partir de los 90 días, el hormigón con CV

Tabla 6

Valores promedio de la influencia de los factores

\begin{tabular}{|c|l|c|c|c|c|}
\hline \multirow{2}{*}{} & \multirow{2}{*}{ Factor } & \multicolumn{4}{|c|}{ Cociente S/R } \\
\cline { 2 - 6 } & & Nivel 1 & Nivel 2 & Nivel 3 & Nivel 4 \\
\hline \multirow{3}{*}{7 días } & Bentonita & 15,33 & 15,87 & 15,02 & 15,20 \\
\cline { 2 - 6 } & Cenizas volantes & 17,79 & 16,52 & 14,09 & 13,02 \\
\cline { 2 - 6 } & Humo de sílice & 14,83 & 14,54 & 15,20 & 16,85 \\
\hline \multirow{2}{*}{14 días } & Bentonita & 19,38 & 18,78 & 18,88 & 18,52 \\
\cline { 2 - 6 } & Cenizas volantes & 20,10 & 19,71 & 18,47 & 17,28 \\
\cline { 2 - 6 } & Humo de sílice & 17,54 & 17,72 & 19,65 & 20,64 \\
\hline \multirow{3}{*}{28 días } & Bentonita & 21,07 & 21,03 & 20,47 & 20,88 \\
\cline { 2 - 6 } & Cenizas volantes & 22,58 & 21,41 & 20,84 & 18,62 \\
\cline { 2 - 6 } & Humo de sílice & 19,28 & 20,52 & 20,75 & 22,90 \\
\hline
\end{tabular}

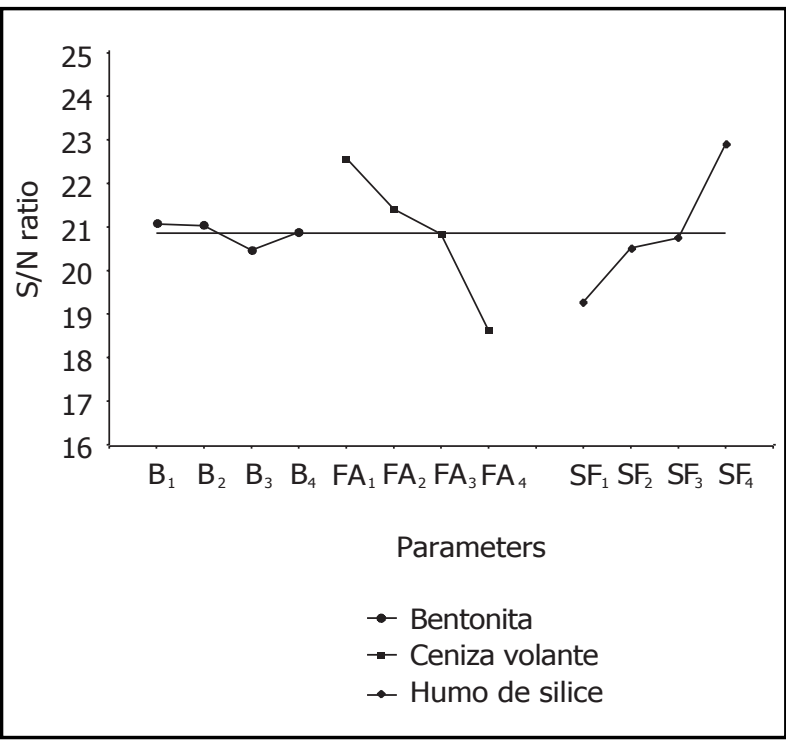

Figura 3. Cociente $S / R$ en función del nivel de los factores a los 28 días.

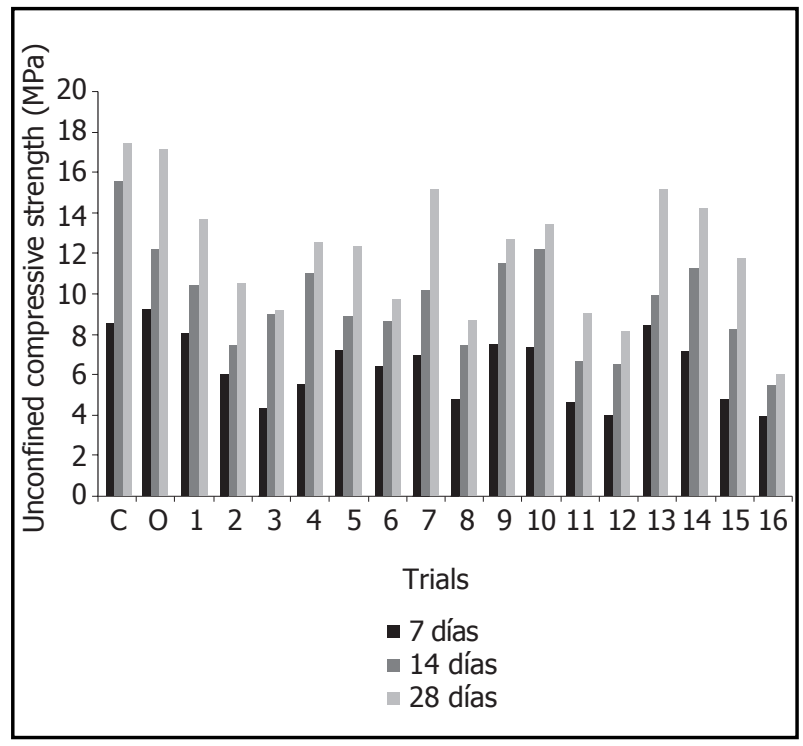

Figura 4. Resistencia a compresión a los 7, 14 y 28 días. 
Tabla 7

Resultados de las análisis de la varianza (ANOVA)

\begin{tabular}{|c|c|c|c|c|c|c|}
\hline & Factores & $\begin{array}{c}\text { Grado de } \\
\text { libertad (GL) }\end{array}$ & $\begin{array}{l}\text { Desviación } \\
\text { por mínimos } \\
\text { cuadrados } \\
\text { (SS) }\end{array}$ & $\begin{array}{c}\text { Varianza } \\
\text { (V) }\end{array}$ & $\begin{array}{c}\text { Razón } \\
\text { de varianzas } \\
\text { F }\end{array}$ & P (\%) \\
\hline \multirow[t]{5}{*}{7 días } & Bentonita & 3 & 1,61 & 0,54 & 0,71 & 2,1 \\
\hline & Cenizas volantes & 3 & 57,40 & 19,13 & 25,49 & 75,2 \\
\hline & Humo de sílice & 3 & 12,83 & 4,28 & 5,70 & 16,8 \\
\hline & Descon. & 6 & 4,503 & 0,75 & & 5,9 \\
\hline & Total & 15 & 76,37 & & & 100,00 \\
\hline \multirow[t]{5}{*}{14 días } & Bentonita & 3 & 1,56 & 0,52 & 0,24 & 2,5 \\
\hline & Cenizas volantes & 3 & 19,67 & 6,56 & 2,98 & 31,8 \\
\hline & Humo de sílice & 3 & 27,37 & 9,12 & 4,15 & 44,3 \\
\hline & Descon. & 6 & 13,20 & 2,2 & & 21,1 \\
\hline & Total & 15 & 61,80 & & & 100 \\
\hline \multirow[t]{2}{*}{28 días } & Bentonita & 3 & 0,89 & 0,30 & 0,12 & 1,2 \\
\hline & Cenizas volantes & 3 & 43,6 & & & \\
\hline
\end{tabular}

Tabla 8

Condiciones óptimas y valores esperados

\begin{tabular}{|c|c|c|c|c|c|c|}
\hline \multirow{2}{*}{$\begin{array}{c}\text { Edad } \\
\text { (días) }\end{array}$} & B & FA & SF & $\begin{array}{c}\text { Valor previsto } \\
\text { (MPa) }\end{array}$ & $\begin{array}{c}\text { Intervalo de } \\
\text { confianza (90\%) } \\
\text { (MPa) }\end{array}$ & $\begin{array}{c}\text { Resultado de } \\
\text { la prueba } \\
\text { de corroboración } \\
\text { (MPa) }\end{array}$ \\
\hline 7 & 0,5 & 10 & 20 & 9,77 & $8,51-11,23$ & 9,25 \\
\hline 14 & 0 & 10 & 20 & 13,10 & $10,30-16,61$ & 12,20 \\
\hline 28 & 0 & 10 & 20 & 17,44 & $13,55-22,45$ & 17,10 \\
\hline
\end{tabular}

presenta una resistencia a compresión mayor que el carece de este aditivo (12).

La finura extrema y la reacción puzolánica del HS tienden a aumentar la resistencia a compresión de la lechada. Al contrario, otras características del HS (como el contenido de carbono y la cantidad que se añade a la lechada) pueden hacer que disminuya. La adición del HS a las lechadas de cemento aumenta la resistencia a compresión de manera significativa debido al efecto "filler" (reducción del tamaño de los poros) y a las excelentes propiedades puzolánicas del humo de sílice. EI HS consta de partículas muy finas de naturaleza vítrea, siendo sus partículas unas 100 veces más pequeñas que las del cemento. Dichos extrema finura y alto contenido de sílice hacen que el HS sea un material muy puzolánico. Por otra parte, al aumentar la relación líquido/sólido, se alarga el tiempo que se requiere para que el humo de sílice provoque una mejora notable de la resistencia a compresión (1). En el presente trabajo, la relación líquido/sólido era de 0,75 .
La bentonita presenta propiedades fuertemente coloidales, aumentándose su volumen varias veces cuando entra en contacto con el agua y convirtiéndose en un material gelatinoso y viscoso. Debido a sus propiedades especiales (hidratación, aumento de volumen, absorción de agua, viscosidad, plasticidad, tixotropía), la bentonita se utiliza como aditivo en las lechadas de cemento. En la presencia de relaciones líquido/sólido bajas, las lechadas que contienen bentonita presentan valores de resistencia a compresión equivalentes a las de las probetas de referencia ( $\sin \mathrm{B}$ ). Con el aumento de la relación líquido/sólido, la incorporación de la bentonita reduce la resistencia de la lechada. Esto se debe a que su inclusión en la lechada genera una mayor porosidad, sobre todo cuando la relación líquido/sólido es alta (1). Entre las ventajas del empleo de la bentonita como aditivo en las lechadas de cemento se cuentan la disminución del peso, el aumento del volumen, la reducción de la viscosidad, la generación de menos calor durante el curado y su menor coste. Como inconvenientes pueden mencionarse que la disminución de la resistencia mecánica y el aumento de la fisuración. 


\section{CONCLUSIONES}

Los resultados de esta investigación permiten concluir que:

- Según la tabla del ANOVA, los factores que más influyen en la resistencia a compresión son los aditivos CV y HS.

- El aumento del contenido de las CV va acompañado de una disminución notable de la resistencia a compresión. Este aditivo puede emplearse para mejorar las propiedades reológicas y la estabilidad de las lechadas; su incorporación en éstas supone, además, una manera de valorizar este residuo.

- Al aumentar el contenido del HS, crece la resistencia a compresión. El humo de sílice puede usarse para mejorar la resistencia a compresión de las lechadas de cemento y para asegurar la eficacia del cemento inyectado.

- La bentonita apenas influye en la resistencia a compresión. Puede utilizarse para mejorar las características reológicas y la estabilidad de las lechadas, y para un mayor aprovechamiento económico de las mismas, por sus propiedades expansivas.

- Las condiciones óptimas para lechadas de 7 días resultaron ser $0,5 \%$ B, $10 \%$ CV y $20 \%$ HS.

- A los 14 y 28 días, las condiciones óptimas fueron $0 \%$ bentonita, $10 \%$ cenizas volantes y $20 \%$ humo de sílice.

- Se ha demostrado la utilidad del método de Taguchi en la ingeniería geotécnica, concretamente en relación con el diseño de las lechadas.

\section{BIBLIOGRAFÍA}

(1) Huang, W. H.: "Properties of cement-fly ash grouth admıxed with bentonite, silica fume, or organic fiber", Cem. Concr. Res., 27, 3 (1997), pp. 395-406.

(2) Allan, M. L.: "Rheology of latex-modified grouts", Cem. Concr. Res., 27, 12 (1997), pp. 1875-1884.

(3) Tan, O. y Zaimoglu, A. S.: "Taguchi approach for investigation of the setting times on cement-based grouts", Indian Journal of Engineering and Materials Sciences, vol. 11 (2003), pp. 63-67.

(4) Tan, O.; Zaimoglu, A. S.; Hinislioglu, S. y Altun, S.: "Taguchi approach for optimization of the bleeding on cement-based grouts", Tunnelling and Underground Space Technology, Volume 20, Issue 2 (2005), pp. 167-173.

(5) Zaimoglu, A. S.: "Bentonit ucucu kul ve silis dumani katkili cimento enjeksiyonu karisimlarinda fiziksel ve mekanik özelliklerin Taguchi metodu ile incelenmesi" (in Turkish), Ph.D. Thesis, Ataturk University, Erzurum (Turkey), 2003.

(6) ASTM standards: Standard test method for compressive strength of hydraulic cement mortars, Designation: C 109, (1998), pp. 71-75.

(7) Taguchi, G.: Tables for Orthogonal arrays and linear graphs, Maruzen, Tokyo, 1962.

(8) Ross, P. J.: Taguchi techniques for quality engineering, MC Graw-Hill, 1987.

(9) Roy, R. K.: A Primer on the Taguchi Method, Competitive Manufacturing Series, 1990.

(10) Huang, W. H.: "Improving the properties of cement-fly ash grout using fiber and superplasticizer", Cem. Concr. Res., 31 (2001), pp. 1033-1041.

(11) Mirza, J.; Mirza, M. S.; Roy, V. y Saleh, K.: "Basic rheological and mechanical properties of high-volume fly ash grouts", Construction and Building Materials, 16 (2002), pp. 353-363.

(12) Lane, R. O. y Best, J. F.: "Properties and use of fly ash in Portland cement concrete", Concrete International, ACI (1982), pp. 81-92. 